\title{
Preons, Gravity and Black Holes
}

\author{
Risto Raitio \\ Independent Researcher, Espoo, Finland \\ Email: risto.raitio@gmail.com
}

How to cite this paper: Raitio, R. (2017) Preons, Gravity and Black Holes. Open Access Library Journal, 4: e3784. https://doi.org/10.4236/oalib.1103784

Received: June 28, 2017

Accepted: July 21, 2017

Published: July 24, 2017

Copyright $\odot 2017$ by author and Open Access Library Inc.

This work is licensed under the Creative Commons Attribution International License (CC BY 4.0).

http://creativecommons.org/licenses/by/4.0/

\section{Open Access}

\begin{abstract}
A previous preon model for the substructure of the standard model quarks and leptons is completed to provide a model of Planck scale gravity and black holes. Gravity theory with torsion is introduced in the model. Torsion has been shown to produce an axial-vector field coupled to spinors, in the present case preons, causing an attractive preon-preon interaction. This is assumed to be the leading term of UV gravity. The boson has an estimated mass near the Planck scale. At high enough density it can materialize and become the center of a black hole. Chiral phase preons are proposed to form the horizon with thickness of order of Planck length. Using quantum information theoretic concepts this is seen to lead to an area law of black hole entropy.
\end{abstract}

\section{Subject Areas}

Particle Physics

\section{Keywords}

Preons, Black Holes, Gravity, Torsion

\section{Introduction}

The purpose of this brief note is to develop further a spin $1 / 2$ preon model in order to give it group theoretic structure, including internal $S U(N)$ type, topological global knot and spacetime Poincaré symmetries. This is an early milestone in a long term process to find a physically acceptable and comprehensive realization for the original model [1]. The model should fulfill four requirements:

1. provide a single global group structure for preons, quarks and leptons,

2. introduce preon properties so that they imply in the adjoint representations the standard model (SM) local gauge group structure $S U(3) \times S U(2) \times U(1)$,

3. introduce Planck scale gravity that is Einstein-Hilbert (EH) compatible, i.e. 
it should contain general relativity (GR) plus corrections in the low energy limit, with least order derivative terms in the action, and

4. propose a tentative corpuscular structure for black holes with an area law entropy of the form $S=\frac{1}{4} A$.

These goals are approached as follows. The preon model [1] [2] [3] [4] can be formulated group theoretically using the results derived by Finkelstein [5]: the global knot algebra $S L q(2)$ symmetry for preons, quarks and leptons. Secondly, the specific construction of the preon model, indicated in (2.1), directly suggests the gauge group structures $S U(2)$ and $S U(3)$ for the weak and strong interactions, respectively.

Thirdly, fermion fields in Einstein-Cartan [6], or Einstein-Cartan-KibbleSciama (ECKS) [7] [8] gravity have been shown by Fabbri to yield interesting results for torsion coupling to spinor fields [9]. This interaction has been shown to express a massive axial-vector field $\mathrm{W}$ coupled to Dirac fermions. The axialvector is used as the force field for preon-preon interactions. It originates from translation, or rather translational rotation, symmetry of the full Poincaré gauge group of the action. A model for Gedanken preon phenomenology is in this way introduced for energy scales, say approximately $10^{16} \mathrm{GeV} \leq E \leq 10^{19} \mathrm{GeV}$. At these energies the axial-vector boson may materialize due to preon-antipreon annihilation in stellar collisions or in similar energy density thermal environment at big bang. At $E \geq 10^{19} \mathrm{GeV}$ the axial-vector bosons may serve as seeds for non-singular black hole formation. Near and above Planck scale the particle interactions due to curvature of general relativity are assumed to be comparatively small, but the essence of GR to spacetime structure rules as traditionally thought. At terrestrial and astronomical scales the effects of curvature dominate gravitational phenomena. There is duality between the standard model particles and black holes. In principle, one is calculable from the other. This is more like Regge-resonance duality rather than the more modern holographic duality. Cursory derivation of the area law for black holes, $S=A / 4$, is provided using quantum information theoretic concepts [10].

The organization of this note is the following. The preon model is described in Section 2. The particle classification group $S L q(2)$ is discussed in Subsection 2.2. The model for black hole structure using the torsion field is described in Subsection 3.1. ECKS gravity with torsion is introduced and summarized in Subsections 3.2 and 3.3. Information theory, correlations and area law are sketched in Section 4. In Section 5 some interesting thoughts on the nature of quantum spinor field equations are briefly quoted. Finally, conclusion are made in Section 6. The Subsections 2.2 and 3.3 can be omitted on first reading.

This note is mostly of mini-review nature with some new proposals for black holes. I do not expect everything to be correct, or even less complete-the goal is achieved if 1) the reductionistic path defined in Subsection 2.1 will be substantiated, and 2) the ECKS theory results as presented here will provide a first approximation for gravity near Planck scale. 


\section{Preons}

\subsection{The Standard Model Particles}

The constituents of quarks and leptons must include an odd number of spin 1/2 particles. I consider the case of three constituents, preons. Requiring charge quantization $\{0,1 / 3,2 / 3,1\}$ and fermionic permutation antisymmetry for same charge preons, four bound states of three light preons have been defined. These form the first generation quarks and leptons consisting (denoted by := below) of preons as follows [1] [2]

$$
\begin{aligned}
& u_{k}:=\epsilon_{i j k} m_{i}^{+} m_{j}^{+} m^{0} \\
& \bar{d}_{k}:=\epsilon_{i j k} m^{+} m_{i}^{0} m_{j}^{0} \\
& e:=\epsilon_{i j k} m_{i}^{-} m_{j}^{-} m_{k}^{-} \\
& \bar{v}:=\epsilon_{i j k} \bar{m}_{i}^{0} \bar{m}_{j}^{0} \bar{m}_{k}^{0}
\end{aligned}
$$

where $m^{+}$is the charge $1 / 3$ preon, $m^{0}$ the neutral preon and $m^{-}$and $\bar{m}^{0}$ are their antiparticles.

A feature in (2.1) with two same charge preons is that the construction provides a three-valued index, which fits well for quark $S U$ (3) color, as it was originally discovered historically [11]. The corresponding color gauge bosons are in the adjoint representation. The weak $S U(2)$ left-handed doublets can be read from the first two and last two lines in (2.1). The standard model gauge structure $S U(N), N=1,2$ is emergent in this sense from the present preon model. In the same way quark-lepton transitions between lines $1 \leftrightarrow 3$ and $2 \leftrightarrow 4$ in (2.1) are possible. The preon and SM fermion group structure is better illuminated with the representations of the $S L q(2)$ group in the subsection 2.2. The above gauge picture is supposed to hold in the present scheme up to the energy of about $10^{16} \mathrm{GeV}$. The electroweak interaction is in the spontaneously broken symmetry phase below energies of the order of $100 \mathrm{GeV}$ and in the symmetric phase above it. The electromagnetic and weak forces take separate ways at higher energies ( $\left.100 \mathrm{GeV} \ll E \ll 10^{16} \mathrm{GeV}\right)$. The weak interaction restores its symmetry but melts away due to ionization of quarks and leptons into preons. The electromagnetic interaction, in turn, stays strong towards Planck scale, $M_{\mathrm{Pl}} \sim 1.22 \times 10^{19} \mathrm{GeV}$. Likewise, the quark color and leptoquark interactions suffer the same destiny as the weak force. One is left with the electromagnetic and gravitational forces only at Planck scale.

The problem of three generations of quarks and leptons is not solved here. Without a true theory of quantum gravity one may just expect that gravitational or topological effects should come into play, but also group theoretic solutions have been proposed in the past.

\subsection{Knot Theory}

Early work on knots in physics goes back in time to 19th and 20th century [12] [13]. On the 21st century Finkelstein has proposed a model based on the group $S L q(2)$ [5]. This group actualizes the needs of the model of the previous 


\section{Section 2 .}

Let us consider the simple case of two dimensional representation of the group $S L q(2)$ which is defined by the matrix

$$
T=D_{m m^{\prime}}^{1 / 2}=\left(\begin{array}{ll}
a & b \\
c & d
\end{array}\right)
$$

where $(a, b, c, d)$ satisfy the knot algebra

$$
\begin{array}{llll}
a b=q b a & b d=q d b & a d-q b c=1 & b c=c b \\
a c=q c a & c d=q d c & d a-q_{1} c b=1 & q_{1} \equiv q^{-1}
\end{array}
$$

where $q$ is defined as follows from the matrix $\epsilon$

$$
\epsilon=\left(\begin{array}{cc}
0 & \alpha_{2} \\
-\alpha_{1} & 0
\end{array}\right)
$$

The matrix $\epsilon$ is invariant under the transformation

$$
T \epsilon T^{t}=T^{t} \epsilon T=\epsilon
$$

where $T^{t}$ is $T$ transposed and $q=\alpha_{1} / \alpha_{2}$.

Higher representations of $S L q(2)$ are obtained by transforming the $(2 j+1)$ monomials

$$
\Psi_{m}^{j}=N_{m}^{j} x_{1}^{n_{+}} x_{2}^{n_{-}},-j \leq m \leq j
$$

by

$$
\begin{aligned}
& x_{1}^{\prime}=a x_{1}+b x_{2} \\
& x_{2}^{\prime}=c x_{1}+d x_{2}
\end{aligned}
$$

where $(a, b, c, d)$ satisfy the knot algebra (2.3) but $x_{1}$ and $x_{2}$ commute and $n_{ \pm}=j \pm m$, and

$$
N_{m}^{j}=\left[\left\langle n_{+}\right\rangle_{q_{1}} !\left\langle n_{-}\right\rangle_{q_{1}} !\right]^{-1 / 2}
$$

and $\langle n\rangle_{q}=\frac{q^{n-1}}{q-1}$. It is found that

$$
\left(\Psi_{m}^{j}\right)^{\prime}=\sum D_{m m^{\prime}}^{j} \Psi_{m^{\prime}}^{j}
$$

where

$$
D_{m m^{\prime}}^{j}(q \mid a, b, c, d)=\sum_{\substack{\delta\left(n_{a}+n_{b}, n_{+}\right) \\ \delta\left(n_{c}+n_{d}, n_{-}\right)}} A_{m m^{\prime}}^{j}\left(q, n_{a}, n_{c}\right) \delta\left(n_{a}+n_{b}, n_{+}^{\prime}\right) a^{n_{a}} b^{n_{b}} c^{n_{c}} d^{n_{d}}
$$

where $n_{ \pm}^{\prime}=j \pm m^{\prime}, D_{m m^{\prime}}^{j}$ is a $2 j+1$ dimensional representation of the $S L q(2)$ algebra and the $A_{m m^{\prime}}^{j}$ is

$$
A_{m m^{\prime}}^{j}\left(q, n_{a}, n_{c}\right)=\left[\frac{\left\langle n_{+}^{\prime}\right\rangle_{1}\left\langle n_{-}^{\prime}\right\rangle_{1}}{\left\langle n_{+}\right\rangle_{1}\left\langle n_{-}\right\rangle_{1}}\right]^{1 / 2} \frac{\left\langle n_{+}\right\rangle_{1} !}{\left\langle n_{a}\right\rangle_{1} !\left\langle n_{b}\right\rangle_{1} !} \frac{\left\langle n_{-}\right\rangle_{1} !}{\left\langle n_{c}\right\rangle_{1} !\left\langle n_{d}\right\rangle_{1} !}
$$

The oriented 2-dimensional projection of a 3-dimensional knot can be assigned three coordinates $(N, w, r)$ where $N$ is the number of crossings, $w$ is the writhe and $r$ the rotation. One can transform to new coordinates $\left(j, m, m^{\prime}\right)$. These indices label the irreducible representations of $D_{m m^{\prime}}^{j}$ of the symmetry 
algebra of the knot, $S L q(2)$ by setting

$$
j=N / 2, m=w / 2, m^{\prime}=(r+o) / 2
$$

This linear transformations makes half-integer representations possible. The knot constraints require $w$ and $r$ to be of opposite parity, therefore $o$ is an odd integer. The knot $(N, w, r)$ may be labeled by $D_{w / 2,(r+o) / 2}^{N / 2}(a, b, c, d)$.

One assigns physical meaning to the $D_{m m^{\prime}}^{j}$ in (2.11) by interpreting the a, b, $c$, and $\mathrm{d}$ as creation operators for spin $1 / 2$ preons. These are the four elements of the fundamental $j=1 / 2$ representation $D_{m m^{\prime}}^{1 / 2}$ as indicated in Table 1. For notational clarity, I use in Table 1 and Table 2. the preon names of [5]. The preon dictionary from the notation of [1] is the following:

$$
\begin{aligned}
& m^{+} \mapsto a, m^{0} \mapsto c \\
& m^{-} \mapsto d, \bar{m}^{0} \mapsto b
\end{aligned}
$$

The standard model particles are the following elements of the $D_{m m^{\prime}}^{3 / 2}$ representation as indicated in Table 2.

All details of the $S L q(2)$ extended standard model are discussed in the review article [5], including the gauge and Higgs bosons and a candidate for dark matter. I do not, however, see much advantage for introducing composite gauge bosons in the model. Introduction of color from preons is done slightly differently in [5]. In the early universe developments there is similarity between the knot model and the present preon model. Therefore, apart from the differences in color interpretation, the model of [1] and the knot algebra of [5] are equivalent in the fermion sector.

In summary, knots having odd number of crossings are fermions and knots with even number of crossings are correspondingly bosons. The leptons and quarks are the simplest quantum knots, the quantum trefoils with three crossings and $j=3 / 2$. At each crossing there is a preon. The free preons are

Table 1. The $D^{1 / 2}$ representation of the four preons.

\begin{tabular}{ccc}
\hline$m$ & $m^{\prime}$ & preon \\
\hline $1 / 2$ & $1 / 2$ & $\mathrm{a}$ \\
$1 / 2$ & $-1 / 2$ & $\mathrm{~b}$ \\
$-1 / 2$ & $1 / 2$ & \multicolumn{2}{c}{} \\
$-1 / 2$ & $-1 / 2$ & $\mathrm{c}$ \\
\hline
\end{tabular}

Table 2. The $D^{3 / 2}$ representation of the standard model particles.

\begin{tabular}{cccc}
\hline$m$ & $m^{\prime}$ & particle & preons \\
\hline $3 / 2$ & $3 / 2$ & electron & aaa \\
$3 / 2$ & $3 / 2$ & neutrino & ccc \\
$3 / 2$ & $-1 / 2$ & d-quark & abb \\
$-3 / 2$ & $-1 / 2$ & u-quark & cdd \\
\hline
\end{tabular}


twisted loops with one crossing and $j=1 / 2$. The $j=0$ states are simple loops with zero crossings.

\section{Gravity}

\subsection{Black Hole Structure}

The Einstein-Hilbert theory of gravity, or general relatitvity, provides rotational curvature (cf. rolling a piece of dough) to spacetime in terms of the metric tensor. This is the prevalent dogma in gravity. It is not, however, the most general case of gauge symmetry available. The EH theory can be generalized by including in the action terms of torsion, which leads to translational curvature (cf. turning screw) in spacetime. This way the full symmetry of the ten parameter Poincaré group can be taken into account. The new theory is called Einstein-CartanKibble-Sciama theory of gravity. Torsion is physically realized by the appearance of an axial-vector boson $W$ (weak interaction bosons are not considered in this note). Interesting enough, the $W$ does not couple to the metric or gauge degrees of freedom [9]. Metric tensor, or gravitons, and torsion are independent degrees of freedom in ECKS gravity, therefore they couple independently to matter.

Preons, with some small value of mass, interact by coupling to the axial-vector boson $W$ with strength $g_{W}$. The preon-preon interaction is attractive [9] providing the binding for three preon states. The mass of the axial-vector boson is estimated to be of the order of the GUT or Planck scale $10^{16-19} \mathrm{GeV}$ (see below in this section). This makes the torsion interaction range very short. At all scales the $W$ couples to preons relatively strongly but to the standard model particles always weakly. I make the proposal that the torsional interaction gives the dominant semi-quantum contribution of gravity at Planck scale. The graviton interaction there is assumed negligible. In the case of black holes, the role of rotational curvature, or gravitons, at Planck scale is traditionally to give the radius-mass relation of the black hole horizon. Below I propose an alternative, dynamical definition of horizon. The $\mathrm{BH}$ singularity is not discussed here except that it is not important within the scope of this note or it is removed by quantum effects.

The field equation for torsion axial-vector is (3.24), from Subsection 3.3

$$
\nabla_{\rho}(\partial W)^{\rho \mu}+M^{2} W^{\mu}=g_{W} \bar{\psi} \gamma^{\mu} \pi \psi
$$

where $M$ is the axial-vector mass, $g_{W}$ the preon--axial-vector coupling and $\psi$ the preon wave function. The coupling $g_{W}$ must be larger than the electromagnetic coupling $\alpha$ to keep the charged preons bound. In ECKS gravity, $g_{W}$ is independent of the gravitational coupling [9]. The key point of this model of gravity is that (3.1) depends only on the axial-vector $W$ and preon $\psi$ fields, not on gauge and metric factors.

Couplings in GUT theory are of the order 0.02 at the GUT scale. With a Yukawa potential in the Schrödinger equation $V(r)=-V_{0} \exp (-a r) / r$ [14], or in our notation $-g_{W} \exp (r / M) / r$ with the physicality condition

$n+l+1 \leq \sqrt{g_{W} m M}$, one may estimate that large $M$ correlates with small preon 
mass $m \ll m_{\text {proton }}$. These matters deserve naturally quantitative attention.

The axial-vector field is expected to appear as a physical particle whenever its production is energetically possible. At Planck scale energy the axial-vector boson serves as a seed for black hole formation causing a black hole to appear. With the growing black hole mass the fermion spins average out towards zero and torsion vanishes but the physical "fat" boson remains.

The horizon may as well be a very thin shell of massless preon-antipreon pairs. In fact, an alternative definition for the horizon can be this dynamical formation of an preon-antipreon "cloud". In that case the nature of gravity near Planck scale would be different from textbook GR. The number of pairs is correlated with the mass of the black hole, and they may form Cooper pairs. A prototype for the lightest black hole is a preon-antipreon- $W$ bound state, see Figure 1. It is a physical state which couples to quark-antiquark and lepton-antilepton pairs. This state was called gravon in [15].

For the local energy density of the gravitational field there is no well-defined covariant notion. Quasilocal energy (QLE) is the next best thing, the energy contained on a two dimensional surface. For round spheres in spherically symmetric spacetimes there is good agreement as to what QLE should be. The standard definition is the classical Misner-Sharp (MS) energy [16] [17] [18].

A good contender with numerous attractive features is the Brown-York (BY) QLE [19] which is based on the covariant Hamilton-Jacobi formulation of general relativity. In [20] the BY QLE was considered in a model where the energy was calculated as coming from elementary surface constituents $E=a /(8 \pi G) A$ where $a$ is the constant proper acceleration of an observer on the stretched horizon and $A$ is the area of the horizon. Therefore black hole energy should be quantized as follows

$$
E=\text { const } \times n \times l_{\mathrm{Pl}}^{2}
$$

with the constant being $a /(8 \pi G)$ and $n=2,3,4, \cdots$ provided a sphere and other polyhedra-type objects can be smoothly covered in the given spacetime with $n$ Planck areas. For comparison, loop quantum gravity gives the following area eigenvalues $A=$ const $\times l_{\mathrm{Pl}}^{2} \sum_{p} \sqrt{j_{p}\left(j_{p}+1\right)}$ with $j_{p}$ a half integer [21].

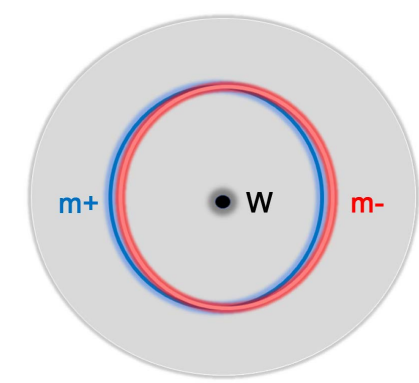

Figure 1. An artist's view of a black hole hypothetical model structure. Blue circle is a preon $m^{+}\left(m^{0}\right)$ and red $m^{-}\left(\bar{m}^{0}\right)$ an anti-preon. $\mathrm{W}$ is the axial-vector boson. The preon-antipreon pair makes the horizon. The axial-vector boson replaces the singularity at the center of the hole in this model. The particles shown are the "partons" of the model. Blurred lines and dot indicate quantum particles. 
The density of a minimal mass black hole inside a sphere of Schwarzschild radius is $10^{-5} / 10^{-99} \mathrm{~g} / \mathrm{cm}^{3}=10^{94} \mathrm{~g} / \mathrm{cm}^{3}$. The density of nuclear matter is a way below this, about $4 \times 10^{14} \mathrm{~g} / \mathrm{cm}^{3}$. In terms of solar mass the density of a black hole is $2 \times 10^{16} \times\left(M_{\text {sun }} / M\right)^{2} \mathrm{~g} / \mathrm{cm}^{3}$ where $M$ is the mass of a star. The torsion induced interaction becomes effective only at densities of matter closer to Planck density than nuclear density, or for objects whose mass is closer to Planck mass rather than the solar mass.

There is in this model a particle-black hole duality in four dimensions. It is Regge-resonance type rather than of the more modern holographic. On the particle side the fermions-quarks and leptons having preons inside-show up. The axial-vector binds the preons, and the graviton is "visible in the sky"starting from GPS systems up to the whole universe. On the black hole side EH gravity provides the shape of spacetime like the wormhole and the horizon where the preons are hidden. The axial-vector is the dominant interaction while those due to gravitons are negligible. In principle, one side is calculable from the other. This duality may help to give the reason to the magical relationship "ER = EPR" [22] (to which I hope to return later).

One may now propose that, as far as there is an ultimate unified field theory, it is a preon theory, like the one proposed here, with Einstein-Cartan-KibbleSciama gravitational and electromagnetic interactions only.

In the early universe, the strong and weak forces are generated only after massless preons combine into quarks and leptons at lower temperature. These two forces function only with short range within nuclei making atoms, molecules and chemistry possible. In a contracting phase of the universe the same processes take place in the reverse order. Massless preon models are a candidate for building conformal cosmological models, see e.g. [23].

\subsection{Einstein-Cartan-Kibble-Sciama Gravity}

To build a full Poincaré group gauge theory for gravity one has boosts, rotations and translations to consider: the rotations lead to curvature and the translations to torsion in spacetime. From a different point of view, curvature arises in the form of metric from energy and torsion in the form of a connection from spin. Torsion is therefore defined on microscopic scales. Torsion requires extension of the Riemann geometry to Riemann-Cartan (RC) geometry [6]. RC gravity, or Einstein-Cartan-Kibble-Sciama (ECKS) [7] [8] gravity can be reduced to Einstein gravity plus torsional contributions. The ECKS theory with Dirac spinor matter fields has been developed by Fabbri [9]. This work yields a massive axial-vector coupled to spinors. His goal in general is to explain most of the open problems in the standard model of particles (and cosmology) as well as to analyze the nature of spinor fields. Here I mainly apply the axial-vector coupling of [9] to preon interactions.

In general relativity metric is used to measure distances and angles. Connections are used to define covariant derivatives. In general form, a covariant derivative of a vector is defined by 


$$
D_{\alpha} V^{\mu}=\partial_{\alpha} V^{\mu}+V^{\rho} \Gamma_{\rho \alpha}^{\mu}
$$

The connection $\Gamma_{\rho \alpha}^{\mu}$ has three indices: $\mu$ and $\rho$ shuffle, or transform, the components of the vector $V^{\rho}$ and $\alpha$ indicates the coordinate in the partial derivative.

Metric and connection should be unrelated. This is implemented by demanding that the covariant derivative of the metric vanishes. In this case the connection is metric-compatible. Metric-compatible connections can be divided into antisymmetric part, given by the torsion tensor, and symmetric part which includes a combination of torsion tensors plus a symmetric, metric dependent connection called the Levi-Civita connection.

In a general Riemannian spacetime $\mathbf{R}$, at each point $p$ with coordinates $x^{\mu}$, there is a Minkowski tangent space $\mathbf{M}=T_{p} \mathbf{R}$, the fiber, on which the local gauge transformation of the $T_{x^{\mu}} \mathbf{R}$ coordinates $x^{a}$ takes place

$$
x^{\prime a}=x^{a}+\epsilon^{a}\left(x^{\mu}\right)
$$

where $\epsilon^{a}$ are the transformation parameters, $\mu$ is a spacetime index and $a$ a fiber frame index.

The dynamics of the theory is based on vierbeins (tetrads) $e_{\mu}^{a}$, not on the metric tensor $g_{\mu \nu}$. The Cartan connection has a primary role and it is

$$
\Gamma_{\mu \lambda v}=e_{\mu}^{a} \partial_{\lambda} e_{a v}
$$

The tensor associated with this connection is torsion tensor

$$
T_{\lambda v}^{\mu}=e_{a}^{\mu}\left(\partial_{\lambda} e_{v}^{a}-\partial_{v} e_{\lambda}^{a}\right)
$$

Unfortunate for the development of gravitation theory, spin was not discovered in the laboratory before 1916. Spinors were introduced in mathematics by Cartan in the 1920's and spinor wave equation was found by Dirac in 1928.

\subsection{Torsion as Axial-Vector Massive Field}

Torsion has the property that it can be separated from gauge and metric factors. Let us start from the metric connection

$$
\Lambda_{\alpha \beta}^{\rho}=\frac{1}{2} g^{\rho \mu}\left(\partial_{\beta} g_{\alpha \mu}+\partial_{\alpha} g_{\mu \beta}-\partial_{\mu} g_{\alpha \beta}\right)
$$

The torsion tensor is completely antisymmetric only if some restrictions are imposed, called the metric-hypercompatibility conditions [24] [25] [26] [27] [28]. Then it can be written in the form

$$
Q_{\alpha \sigma v}=\frac{1}{6} W^{\mu} \varepsilon_{\mu \alpha \sigma v}
$$

where $W^{\mu}$ is torsion pseudo-vector, obtained from the torsion tensor after a Hodge dual. With the metric connection and the torsion pseudo-vector the most general connection can be written as a sum of $\Lambda_{\alpha \beta}^{\rho}$ and $Q_{\alpha \sigma v}$ as follows

$$
\Gamma_{\alpha \beta}^{\rho}=\frac{1}{2} g^{\rho \mu}\left[\left(\partial_{\beta} g_{\alpha \mu}+\partial_{\alpha} g_{\mu \beta}-\partial_{\mu} g_{\alpha \beta}\right)+\frac{1}{6} W^{v} \varepsilon_{\nu \mu \alpha \beta}\right]
$$

Functions $\Omega_{b \mu}^{a}$ that transform under a general coordinate transformation 
like a lower Greek index vector and under a Lorentz transformation as

$$
\Omega_{b^{\prime} v}^{\prime a^{\prime}}=\Lambda_{a}^{a^{\prime}}\left[\Omega_{b v}^{a}-\left(\Lambda^{-1}\right)_{k}^{a}\left(\partial_{v} \Lambda\right)_{b}^{k}\right]\left(\Lambda^{-1}\right)_{b^{\prime}}^{b}
$$

are called a spin connection. The torsion in coordinate formalism is defined as follows

$$
Q_{\mu \nu}^{a}=-\left(\partial_{\mu} e_{v}^{a}-\partial_{\nu} e_{\mu}^{a}+e_{\nu}^{b} \Omega_{b \mu}^{a}-e_{\mu}^{b} \Omega_{b v}^{a}\right)
$$

and the spin connection is given by

$$
\Omega_{b \mu}^{a}=e_{b}^{v} e_{\rho}^{a}\left(\Gamma_{v \mu}^{\rho}-e_{k}^{\rho} \partial_{\mu} e_{v}^{k}\right)
$$

which is antisymmetric in the two Lorentz indices after both of them are brought in the same upper or lower position. The most general spinorial connection is

$$
\boldsymbol{\Omega}_{\mu}=\frac{1}{2} \Omega_{a b \mu} \boldsymbol{\sigma}^{a b}+i q A_{\mu} \mathbb{I}
$$

where $A_{\mu}$ is the gauge potential. The spinorial curvature is using the spinorial connection

$$
\boldsymbol{F}_{\alpha \beta}=\partial_{\alpha} \boldsymbol{\Omega}_{\beta}-\partial_{\beta} \boldsymbol{\Omega}_{\alpha}+\left[\boldsymbol{\Omega}_{\alpha}, \boldsymbol{\Omega}_{\beta}\right]
$$

Let us define the decomposition of the spinor field in its left and right parts

$$
\begin{array}{ll}
\pi_{L} \psi=\psi_{L} & \bar{\psi} \pi_{R}=\bar{\psi}_{L} \\
\pi_{R} \psi=\psi_{R} & \bar{\psi} \pi_{L}=\bar{\psi}_{R}
\end{array}
$$

so that

$$
\bar{\psi}_{L}+\bar{\psi}_{R}=\bar{\psi} \quad \psi_{L}+\psi_{R}=\psi
$$

Now one has 16 linearly-independent bi-linear spinorial quantities

$$
\begin{gathered}
2 \bar{\psi} \sigma^{a b} \pi \psi=\Sigma^{a b} \\
2 i \bar{\psi} \sigma^{a b} \psi=S^{a b} \\
\bar{\psi} \gamma^{a} \pi \psi=V^{a} \\
\bar{\psi} \gamma^{a} \psi=U^{a} \\
i \bar{\psi} \pi \psi=\Theta \\
\bar{\psi} \psi=\Phi
\end{gathered}
$$

To have the most general connection decomposed into the simplest symmetric connection plus torsion terms we substitute (3.9) in (3.12) and this in (3.13). The field equations reduce to the following

$$
\nabla_{\rho}(\partial W)^{\rho \mu}+M^{2} W^{\mu}=g_{W} \bar{\psi} \gamma^{\mu} \pi \psi
$$

for torsion axial-vector and

$$
\begin{aligned}
& R^{\rho \sigma}-\frac{1}{2} \operatorname{Rg}^{\rho \sigma}-\Lambda g^{\rho \sigma}=\frac{k}{2}\left[\frac{1}{4} F^{2} g^{\rho \sigma}-F^{\rho \alpha} F_{\alpha}^{\sigma}+\frac{1}{4}(\partial W)^{2} g^{\rho \sigma}\right. \\
& -(\partial W)^{\sigma \alpha}(\partial W)_{\alpha}^{\rho}+M^{2}\left(W^{\rho} W^{\sigma}-\frac{1}{2} W^{2} g^{\rho \sigma}\right) \\
& +\frac{i}{4}\left(\bar{\psi} \gamma^{\rho} \nabla^{\sigma} \psi-\nabla^{\sigma} \bar{\psi} \gamma^{\rho} \psi+\bar{\psi} \gamma^{\sigma} \nabla^{\rho} \psi-\nabla^{\rho} \bar{\psi} \gamma^{\sigma} \psi\right) \\
& \left.-\frac{1}{2} g_{W}\left(W^{\sigma} \bar{\psi} \gamma^{\rho} \pi \psi+W^{\rho} \bar{\psi} \gamma^{\sigma} \pi \psi\right)\right]
\end{aligned}
$$


for the torsion-spin and curvature-energy coupling, and

$$
\nabla_{\sigma} F^{\sigma \mu}=q \bar{\psi} \gamma^{\mu} \psi
$$

for the gauge-current coupling; and finally

$$
i \gamma^{\mu} \nabla_{\mu} \psi-g_{W} W_{\sigma} \gamma^{\sigma} \pi \psi-m \psi=0
$$

for the spinor field equations which again can be split as

$$
\begin{gathered}
\frac{i}{2}\left(\bar{\psi} \gamma^{\mu} \nabla_{\mu} \psi-\nabla_{\mu} \bar{\psi} \gamma^{\mu} \psi\right)-g_{W} W_{\sigma} V^{\sigma}-m \Phi=0 \\
\nabla_{\mu} U^{\mu}=0 \\
\frac{i}{2}\left(\bar{\psi} \gamma^{\mu} \pi \nabla_{\mu} \psi-\nabla_{\mu} \bar{\psi} \gamma^{\mu} \pi \psi\right)-g_{W} W_{\sigma} U^{\sigma}=0 \\
\nabla_{\mu} V^{\mu}-2 m \Theta=0 \\
i\left(\bar{\psi} \nabla^{\alpha} \psi-\nabla^{\alpha} \bar{\psi} \psi\right)-\nabla_{\mu} S^{\mu \alpha}+2 g_{W} W_{\sigma} \Sigma^{\sigma \alpha}-2 m U^{\alpha}=0 \\
\nabla_{\alpha} \Phi-2\left(\bar{\psi} \sigma_{\mu \alpha} \nabla^{\mu} \psi-\nabla^{\mu} \bar{\psi} \sigma_{\mu \alpha} \psi\right)+2 g_{W} \Theta W_{\alpha}=0 \\
\nabla_{v} \Theta-2 i\left(\bar{\psi} \sigma_{\mu \nu} \pi \nabla^{\mu} \psi-\nabla^{\mu} \bar{\psi} \sigma_{\mu \nu} \pi \psi\right)-2 g_{W} \Phi W_{v}+2 m V_{v}=0 \\
\left(\nabla_{\alpha} \bar{\psi} \pi \psi-\bar{\psi} \pi \nabla_{\alpha} \psi\right)+\nabla^{\mu} \sum_{\mu \alpha}+2 g_{W} W^{\mu} S_{\mu \alpha}=0 \\
\nabla^{\mu} V^{\rho} \varepsilon_{\mu \rho \alpha \nu}+i\left(\bar{\psi} \gamma_{[\alpha} \nabla_{\nu]} \psi-\nabla_{[v} \bar{\psi} \gamma_{\alpha]} \psi\right)+2 g_{W} W_{[\alpha} V_{v]}=0 \\
\nabla^{[\alpha} U^{\nu]}+i \varepsilon^{\alpha \nu \mu \rho}\left(\bar{\psi} \gamma_{\rho} \pi \nabla_{\mu} \psi-\nabla_{\mu} \bar{\psi} \gamma_{\rho} \pi \psi\right)-2 g_{W} W_{\sigma} U_{\rho} \varepsilon^{\alpha v \sigma \rho}-2 m S^{\alpha \nu}=0
\end{gathered}
$$

together equivalent to the spinor field equations above. From (3.24) one sees that torsion behaves like a massive axial-vector field satisfying Proca field equations. It is noted that torsion does not couple to metric or gauge fields. Torsion and $\mathrm{EH}$ gravitation seem to have the same coupling constant. However, in [9] it is shown that using the Einstein-Cartan-Sciama-Kibble field equations these two independent fields with independent sources can have independent coupling constants.

The preon-preon interaction is attractive and of short range due to the mass of the axial-vector field. The interaction includes two free parameters, the coupling constant $g_{W}$ and the mass $M$ of the axial-vector. Therefore, bound states of preons may be formed in principle by the axial-vector interaction.

The ECKS field equation described above can be derived using the standard variational method from a dynamical action whose Lagrangian function (up to possible scalars fields) is given as follows

$$
\begin{aligned}
\mathscr{L}= & -\frac{1}{4}(\partial W)^{2}+\frac{1}{2} M^{2} W^{2}-\frac{1}{k} R-\frac{2}{k} \Lambda-\frac{1}{4} F^{2} \\
& +i \bar{\psi} \gamma^{\mu} \nabla_{\mu} \psi-g_{W} \bar{\psi} \gamma^{\mu} \pi \psi W_{\mu}-m \bar{\psi} \psi
\end{aligned}
$$

The above Lagrangian (3.28) is theoretically sound. But for specific calculations analytic and/or numerical approximations must be developed.

\section{Correlations and Area Law}

Correlations between between two systems, say A and B, are information of A 
about B. Usually correlations are calculated as the decay of two-point functions as a function of distance. In physical situations it is often asked how correlations between a connected region $A$ and its environment $B$ scale with the size of the region $B$. In quantum systems at zero temperature all correlations are due to entanglement which is measured by entropy. The entropy of black holes scales with the area-not volume-of the surface at the event horizon. The area law holds in the form that the maximal information content of the surface comes from elementary areas of size $l_{\mathrm{P} 1}^{2}$ containing one bit of information.

Area law for entropy is found also in non-critical quantum lattice systems whereas critical systems allow for small logarithmic corrections. The area law can be intuitively understood by introducing characteristic length scale, the correlation length, which gives a measure of how fast two-point correlations decay. If the correlation length can be estimated, or calculated, and it is small compared to the typical size of the system, the area law is expected. This cannot, however, be rigorously proved in general.

In the following I make use of the lattice model and results of [10] and introduce a quantum information theory concept, the mutual information. The mutual information and entanglement entropy coincide at zero temperature. Secondly, mutual information measures the total amount of information of one system about another without leaving out hidden correlations. Thirdly, the area law can be proved at finite temperatures.

Consider a system on lattice $L$ in $D$ spatial dimension which is translational invariant. Each lattice site represents a quantum spin state in Hilbert space $\mathbb{C}^{d}$. Assume a probability function $\rho$ on the lattice and $\rho_{A}, \rho_{B}$ corresponding to disjoint sets $A, B \subseteq L$. The mutual information between sets $A$ and $B$ is defined as follows

$$
I(A: B)=S\left(\rho_{A}\right)+S\left(\rho_{B}\right)-S\left(\rho_{A B}\right)
$$

where $S(\rho)=-\operatorname{tr}(\rho \log \rho)$ is von Neumann entropy. The mutual information is the total amount of correlations between two systems. (4.1) quantifies the information about $B$ obtainable from $A$. Properties of mutual information are: positivity, it vanishes if and only if the system factorizes and it is non-increasing under discarding parts of the system.

The correlation length, defined by mutual information, is related with the area law. Consider a spherical shell with outer radius $R$ and wall thickness $L \ll R$. The shell separates the inner region $A$ from the exterior $B$. Denote the mutual information between $A$ and $B$ by $I_{L}(R)$ and define $\xi_{M}$ as the minimal length obeying the inequality

$$
I_{L}(R)<I_{0}(R) / 2
$$

for all $R$. In other words, correlation length is measured by the mutual information. From the subadditivity property of entropy it follows that

$$
\begin{aligned}
& I(A: B C) \leq I(A: B)+2 S_{C} \text { and from this one gets } \\
& \qquad I_{0} \leq I_{\xi_{M}}+2 S_{C} \leq 4|\partial A| \xi_{M}
\end{aligned}
$$


where $\partial A$ is the subset of $\mathrm{A}$ which is connected to the exterior by an interaction. The second inequality is obtained from the first one by inserting $I_{\xi_{M}} \leq 1 / 2 I_{0}$ and the relation $S(C) \leq \xi_{M}|\partial A|$. Therefore one gets an area law for the mutual information from the existence of the length scale $\xi_{M}$ alone. This area law holds for zero temperature, too. Its violation implies infinite correlation length $\xi_{M}$.

Consider now a simple case of one dimensional long lattice. It can be obviously extended to two dimensions with additional translational symmetry. Start with an one dimensional finitely correlated state (FCS) [29]. Each FCS can be described by a completely positive, trace preserving map $T: \mathcal{H}_{1} \rightarrow \mathcal{H}_{1} \otimes \mathcal{H}_{2}$ with $\mathcal{H}_{1}, \mathcal{H}_{2}$ being Hilbert spaces of dimension $D_{1}, D_{2}$, respectively. Introduce $\mathcal{E}(x)=t r_{2}[T(x)]$ and assume that $\mathcal{E}(x)$ has just one eigenvalue of the order of one. The second largest eigenvalue of $\mathcal{E}, \varepsilon_{2}$, depends on the standard correlation length like this: $\xi \sim-1 / \log \varepsilon_{2}$. To get an idea of $\xi_{M}$ consider the fact that $\rho_{A B}$ factorizes exponentially with the separation $L$ [10]

$$
\left\|\rho_{A B}-\rho_{A} \otimes \rho_{B}\right\|_{1}=\mathcal{O}(\exp (-L / \xi))
$$

With a few more steps it is found that

$$
I_{L}(R) \leq \log (D) \mathcal{O}(L \exp (-L / \xi))
$$

Now $I_{L}(R)$ is bound from below, increases with $R$ and decreases with $L$. Therefore $\xi_{M}$ is finite and connected to $\xi$.

Specify next the system as consisting of spin $1 / 2$ singlets. For a given site $i$ the probability of having s singlet at site $j$ is a function $f(|i-j|)$. The number of singlets that connect two regions in the lattice gives the mutual information between those regions. Consider a case with $f(x) \propto \exp (-x / \xi)$, then the following conditions hold: (a) all correlation functions decay exponentially with the diistance and $\xi$ gives the correlation length, (b) $I_{L}(R)$ decays exponentially with $L$ and $\xi_{M} \propto \xi$ and (c) an area law is found. If the probability function is $f(x) \propto 1 /\left(x^{2}+a^{2}\right)$ correlation functions decay as power laws with distance and the area law is violated with infinite correlation length.

\section{Considerations of Spinors Fields}

The incompatibility of gravity and second quantization, as well as the problem of radiative corrections, are discussed from a novel point of view in [9]. A major point is that, with gravity included in the theory, plane wave solutions do not exist any more. Instead, semi-localized fields can be derived by analyzing the self-interactions of the chiral components of the spinor fields.

In quantum theory electrons are point-like particles. Radiative processes involving loop diagrams give the electron a self-interaction leading to mass and charge renormalization. It is proposed in [9] that the self-interaction of the electron field should be the mutual interaction of its two chiral components giving internal dynamics for extended fields. Consequently Zitterbewegung would actually influence the particles. The Zitterbewegung of Dirac spinor fields and quantum effects for structureless particles might coincide. If true this point 
of view would imply a paradigm shift. And we might be closer to quantum gravity than commonly believed.

\section{Conclusions}

The preon model with spin $1 / 2$ and charge 0 and $\pm 1 / 3$ constituents discussed above has a sound group theoretical structure based on some of the most successful groups. Both the preons and the quarks and leptons belong to two lowest representations of the global SLq(2) group, shown in Table 1 and Table 2 in Subsection 2.2. With the four preons the local gauge groups of the standard model, $S U(3) \times S U(2) \times U(1)$ become visible. Preons, as Dirac spinors, are the fundamental building blocks of matter which couple to gravity near Planck scale predominantly by axial-vector boson coupling. Above the Planck scale the formation of black holes becomes possible with the axial-vector boson forming a seed for the hole and the chiral phase preon-antipreon pairs move on the horizon. The axial-vector coupled to the preons may make the singularity of the hole softer or fade away.

It is hoped that the preon scheme [4] would provide a way towards a better understanding of the roles of all interactions. For that goal a special construction is proposed for the weak and strong interactions. They are bona fide gauge theories but emerge from the very basic fermion structure of the model (2.1). Gravity and electromagnetism are the "original" long range interactions in the big bang of cyclic cosmology.

For quantum gravity the essential problem in model building is that we do not know the nature of quantum geometry. And we have no data to guide us. In this note I have taken the attitude that quantum gravity may, after all, be simpler than expected. At least the UV part of it may be closer to present quantum theory than commonly thought. In the present treatment, all the basic equations, of the standard model and the torsion field 3.24, are relativistic quantum equations. Therefore quantum gravity may be within reach in the sense of Section 5. The scenario presented above serves for most practical purposes where gravity is considered: for terrestrial to cosmological distances the classical Einstein-Hilbert equations work well and for the highly elusive Planck scale distances the Proca equation is a natural candidate, yet to be tested.

A dual relationship was proposed between matter and black holes in four dimensions. One is, in principle, calculable from the other. But calculational methods for solving the field Equations (3.24)-(3.27), or the Lagrangian 3.28, are yet to be developed.

The role of rotational curvature of Einstein-Hilbert gravity needs to be quantitatively analyzed in the extended ECKS theory with spinor fields. EH theory is assumed here: 1) to give the horizon and the Einstein-Rosen bridge, or wormhole, in spacetime, but 2) to be a small correction to the torsion caused interactions at Planck scale. It is remarkable that the equations for torsion (3.24) and curvature (3.25) are so different with the torsion being independent of metric and gauge degrees of freedom. Zitterbewegung at Planck energy should 
provide a scale of length/area for calculations.

The translation symmetry of the full Poicaré group implies axial-vector interactions which introduce a new Gedanken phenomenology for preons between the GUT scale and Planck scale. The axial-vector particle is expected to have a large mass, $M \sim 10^{16-19} \mathrm{GeV}$. Within accelerator energies axial-vector particle couplings to standard model particles are very small.

The main contribution of ECKS gravity to the present preon model is that it leads to the Proca equation of the preon spinor field coupled to torsion field 3.24. I tentatively propose the spinor-torsion field coupling as the dominant interaction in the UV limit of quantum gravity. Thus there should be quantum states describing the preon-axial-vector system to which the information theoretic arguments of Section 4 can be applied leading to the area rule $S_{B H}=1 / 4 \mathrm{~A}$.

Of matters not discussed in this note I refer again to [9] where substantial amount of phenomenological success is obtained beyond the standard model of cosmology, like dark matter, cosmological constant and inflation. Thermodynamics is another area to be studied in detail. More work is needed to clarify all the issues and gain consensus in the questions like field quantization, gravity and its full quantum version.

\section{Acknowledgements}

I thank Luca Fabbri for correspondence.

\section{References}

[1] Raitio, R. (1980) A Model of Lepton and Quark Structure. Physica Scripta, 22, 197. https://doi.org/10.1088/0031-8949/22/3/002

[2] Raitio, R. (2016) Combinatorial Preon Model for Matter and Unification. Open Access Library Journal, 3: e3032. https://doi.org/10.4236/oalib.1103032

[3] Raitio, R. (2017) On the Conformal Unity between Quantum Particles and General Relativity. Open Access Library Journal, 4: e3342.

https://doi.org/10.4236/oalib.1103342

[4] Raitio, R. (2017) Preons, Standard Model, Gravity with Torsion and Black Holes. Open Access Library Journal, 4: e3632. https://doi.org/10.4236/oalib.1103632

[5] Finkelstein, R. (2016) On the SLq(2) Extension of the Standard Model and the Measure of Charge. International Journal of Modern Physics A, 32, Article ID: 17300010. https://doi.org/10.1142/S0217751X17300010

[6] Cartan, E. (1980) Cosmology and Gravitation: Spin, Torsion, Rotation, and Supergravity. In: Bergmann, P.G. and de Sabbata, V., Eds., NATO ASIB Proc. 58: Cosmology and Gravitation: Spin, Torsion, Rotation, and Supergravity, 489-491.

[7] Kibble, T. (1961) Lorentz Invariance and the Gravitational Field. Journal of Mathematical Physics, 2, 212. https://doi.org/10.1063/1.1703702

[8] Sciama, D. (1962) In Recent Developments in General Relativity. Oxford.

[9] Fabbri, L. (2017) Foundations Quadrilogy. https://arxiv.org/abs/1703.02287

[10] Wolf, M., Verstraete, F., Hastings, M. and Cirac, J. (2008) Area Laws in Quantum Systems: Mutual Information and Correlations. Physical Review Letters, 100, Article ID: 070502. https://doi.org/10.1103/physrevlett.100.070502 
[11] Greenberg, O. (2009) The Color Charge Degree of Freedom in Particle Physics, Compendium of Quantum Physics Berger. Springer-Verlag, Berlin, 109-111.

[12] Thomson, W. (1868) VI. - On Vortex Motion on Vortex Motion. Transactions of the Royal Society of Edinburgh, 25, 217-260.

https://doi.org/10.1017/S0080456800028179

[13] Faddeev, L. and Niemi, A. (1997) Knots and Particles. Nature, 387, 58-66. https://doi.org/10.1038/387058a0

[14] Hamzavi1, M., Movahedi, M., Thylwe, K.E. and Rajabi, A. (2012) Approximate Analytical Solution of the Yukawa Potential with Arbitrary Angular Momenta. Chinese Physics Letters, 29, Article ID: 080302. https://doi.org/10.1088/0256-307x/29/8/080302

[15] Raitio, R. (2015) The Decay of a Black Hole in a GUT Model. Open Access Library Journal, 2, Article ID: e2031. https://doi.org/10.4236/oalib.1102031

[16] Misner, C. and Sharp, D. (1964) Relativistic Equations for Adiabatic, Spherically Symmetric Gravitational Collapse. Physical Review, 136, B571-B576. https://doi.org/10.1103/physrev.136.b571

[17] Hernandez, W.C. and Misner, C.W. (1966) Observer Time as a Coordinate in Relativistic Spherical Hydrodynamics. The Astrophysical Journal, 143, 452-464. https://doi.org/10.1086/148525

[18] Cahill, M.E. and McVittie, G.C. (1970) Spherical Symmetry and Mass-Energy in General Relativity I. General Theory. Journal of Mathematical Physics, 111382 1391.

[19] Brown, J. and York, J.J. (1993) Quasilocal Energy and Conserved Charges Derived from the Gravitational Action. Physical Review, 47, 1407. https://doi.org/10.1103/physrevd.47.1407

[20] Raitio, R. (2016) Standard Model Matter Emerging from Spacetime Preons. Open Access Library Journal, 3, Article ID: e2788. https://doi.org/10.4236/oalib.1102788

[21] Rovelli, C. (2011) Zakopane Lectures on Loop Gravity. Physics, PoS QGQGS2011, 003 [arXiv/gr-qc:1102.3660].

[22] Maldacena, J. and Susskind, L. (2013) Cool Horizons for Entangled Black Holes. Fortschritte der Physik, 61, 781-811. https://doi.org/10.1002/prop.201300020

[23] Bars, I., Steinhardt, P. and Turok, N. (2013) Cyclic Cosmology, Conformal Symmetry and the Metastability of the Higgs. Physics Letters, B726, 50-55. https://doi.org/10.1016/j.physletb.2013.08.071

[24] Fabbri, L. (2007) On a Completely Antisymmetric Cartan Torsion Tensor. Annales de la Fondation Louis de Broglie, 32, 215.

[25] Hayashi, K. (1976) Restrictions on Gauge Theory of Gravitation. Physics Letters B, B65, 437-440. https://doi.org/10.1016/0370-2693(76)90437-8

[26] Yu, X. (1989) Astrophysical and Space. Science, 154, 321. https://doi.org/10.1007/BF00642814

[27] Audretsch, J. and Lammerzahl, C. (1988) Constructive Axiomatic Approach to Spacetime Torsion. Classical \& Quantum Gravity, 5, 1285. https://doi.org/10.1088/0264-9381/5/10/008

[28] Macias, A. and Lammerzahl, C. (1993) On the Dimensionality of Space-Time. Journal of Mathematical Physics, 34, 4540.

[29] Fannes, M., Nachtergaele, B. and Werner, R. (1992) Finitely Correlated States on Quantum Spin Chains. Communications in Mathematical Physics, 144, 443. https://doi.org/10.1007/BF02099178 
Submit or recommend next manuscript to OALib Journal and we will provide best service for you:

- Publication frequency: Monthly

- 9 subject areas of science, technology and medicine

- Fair and rigorous peer-review system

- Fast publication process

- Article promotion in various social networking sites (LinkedIn, Facebook, Twitter, etc.)

- Maximum dissemination of your research work

Submit Your Paper Online: Click Here to Submit

Or Contact service@oalib.com 